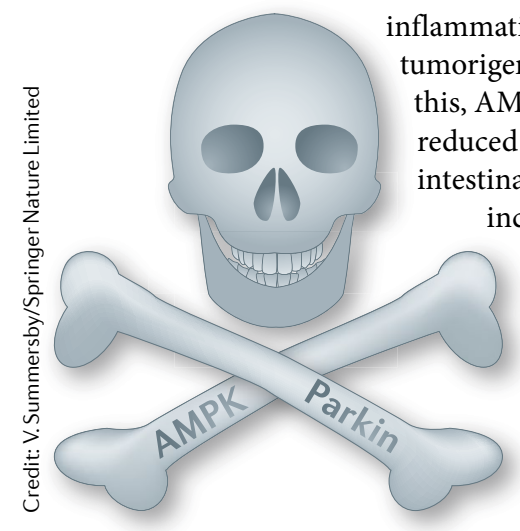

mation-associated umorigenesis. In line with this, AMPK activation educed the number of estinal tumours and increased survival in a mouse model of pharmacologically induced inflammation-related carcinogenesis. Furthermore, the authors found that, in tissues from individuals with colon cancer or inflammatory bowel disease, parkin expression was reduced and negatively correlated with RIPK3 activation.

Moving forwards, targeting the AMPK-parkin-RIPK3 axis opens up new therapeutic possibilities for
AMPKparkin-RIPK3 axis suppresses inflammationassociated tumorigenesis including cancers.

Paulina Strzyz

ORIGINAL ARTICLE Lee, S. B. et al. The AMPKparkin axis negatively regulates necroptosis and tumorigenesis by inhibiting the necrosome. Nat. Cell Biol. 21, 940-951 (2019) RELATED ARTICLES Weinlich, R. et al. Necroptosis in development, inflammation and disease. Nat. Rev. Mol. Cell Biol. 18, 127-136 (2017)

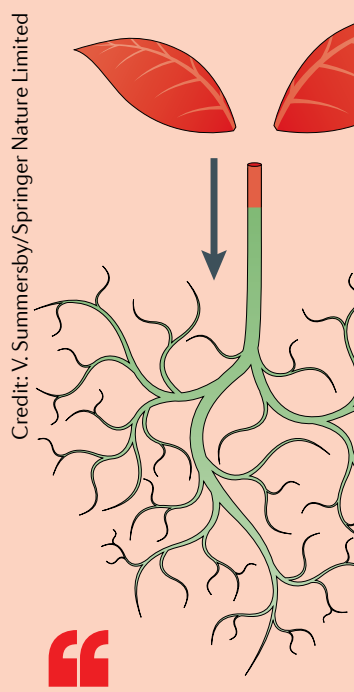

cytosine methylation in the TCTP 1 mRNA coding region is required for mRNA mobility

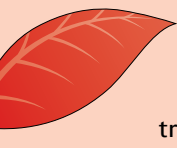

lost mobility in methylation-deficient mutants or plants treated with a methylation inhibitor, confirming that lack of methylation affects specifically mRNA mobility and not phloem-mediated diffusion of small proteins such as YFP. Of note, the loss of mobility was not due to decreased stability of transcripts.

Lastly, TCTP1 mRNA mobility was required for the function of the TCTP1 protein, as the non-mobile mRNA was unable to enhance the growth of grafted roots.

This study establishes the importance of cytosine methylation for mRNA transport in plants, as $m^{5} \mathrm{C}$, alone or in combination with specific sequences (such as PTB), guides mRNAs to specific tissues and cell types.

Kim Baumann

ORIGINAL ARTICLE Yang, L. et al. $\mathrm{m}^{5} \mathrm{C}$ methylation guides systemic transport of messenger RNA over graft junctions in plants. Curr. Biol. https://doi.org/10.1016/j.cub.2019.06.042 (2019)

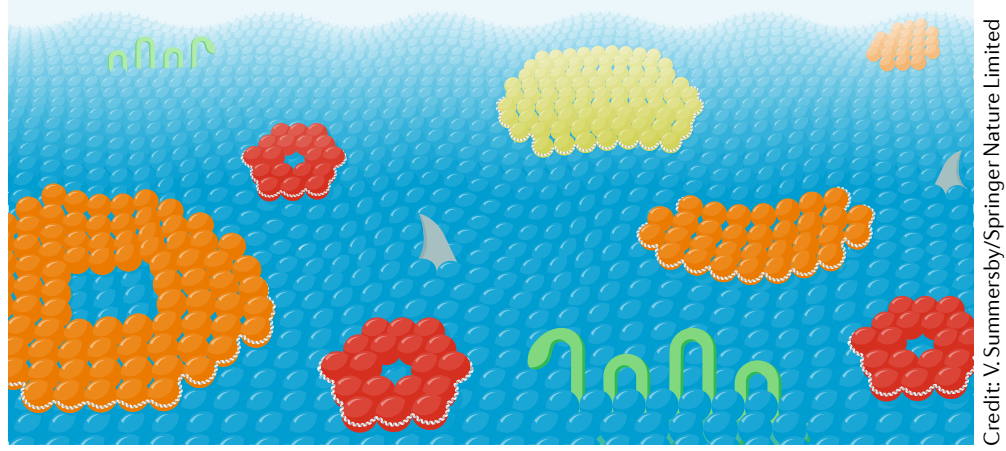

MEMBRANE DYNAMICS

\section{Tying lipid rafts to oncogenic signalling}

Genetic amplifications and gain of function of growth factor signalling are among the most common drivers of cancer. Although it is widely accepted that local plasma membrane organization and lipid composition affect membraneassociated protein function, including signalling receptors, these links are generally poorly understood, and even more so in specific contexts such as cancer. Bi et al. now find that increased synthesis of saturated phospholipids and the formation of ordered lipid membrane domains support oncogenic growth factor signalling.

Lipidomic analysis of a primary glioblastoma U87 cell line indicated that expression of a constitutively active epidermal growth factor receptor (EGFR) variant, EGFRvIII - a common driver of glioblastoma - caused membrane lipid remodelling. Specifically, the levels of saturated phosphatidylcholine (PC) species, produced by the activity of lysophosphatidylcholine acyltransferase 1 (LPCAT1), were increased. This increase was dependent on EGFRvIll signalling, which promoted LPCAT1 expression. In turn, LPCAT1 knockdown suppressed EGFR signalling, which was associated with increased internalization of EGFR from the plasma membrane; EGFR signalling could be restored by supplying saturated PC or its precursors. Thus, oncogenic EGFR signalling drives LPCAT1-mediated synthesis of saturated lipid species, which is required for EGFR residence at the plasma membrane and its sustained signalling.

Saturated membrane lipids promote the formation of ordered domains (lipid rafts). Consistent with its role in stimulating synthesis of saturated PC species, expression of EGFRvIll increased membrane order in U87 cells in a LPCAT1-dependent manner. Furthermore, EGFRvIll partitioned to ordered domains, suggesting that EGFRvIll signalling is augmented by receptor association with lipid rafts.

LPCAT1 depletion reduced cancer cell growth in the U87 cell line and patientderived glioblastoma cells as well as in different cancer cell lines with LPCAT1 amplification (which has been detected in various cancers, in particular, highly aggressive tumours with poor patient prognosis). Moreover, LPCAT1 depletion inhibited growth of glioblastoma and LPCAT1-amplified tumours in mice.

In summary, LPCAT1-mediated membrane lipid saturation and subsequent ordering of the plasma membrane is an important mechanism driving oncogenic signalling and tumour growth. LPCAT1 could now be explored as a potential new drug target in oncology.

Paulina Strzyz

ORIGINAL ARTICLE Bi, J. et al. Oncogene amplification in growth factor signaling pathways renders cancers dependent on membrane lipid remodeling. Cell Metab. https://doi.org/10.1016/j.cmet.2019.06.014 (2019) FURTHER READING Sezgin, E. et al. The mystery of membrane organization: composition, regulation and roles of lipid rafts. Nat. Rev. Mol. Cell Biol. 18, 361-374 (2017) | Harayama, T. \& Riezman, H. Understanding the diversity of membrane lipid composition. Nat. Rev. Mol. Cell Biol. 19, 281-296 (2018) 